\title{
About accidents cyclicality and mathematical model of failure rate in main electrical grids of power systems
}

\author{
Irek Galiaskarov ${ }^{1}$, Misrikhan Misrihkanov², Vladimir Ryabchenko ${ }^{3 *}$ \\ ${ }^{1}$ JSC “CEMC UES”, 4, Belovezhskaya st., Moscow, Russia \\ ${ }^{2}$ National research university "MPEI", Institute of Electrical Power Engineering, 14 Krasnokazarmennaya St, Moscow, Russia \\ ${ }^{3}$ JSC “R\&D Center FGC UES”, Building 3, 22 Kashirskoye Highway, Moscow, Russia
}

\begin{abstract}
The accidents cyclicality of $500 \mathrm{kVoverhead} \mathrm{lines} \mathrm{of} \mathrm{a} \mathrm{large} \mathrm{region} \mathrm{over} \mathrm{an} \mathrm{extended} \mathrm{time} \mathrm{period}$ was studied. Significant fluctuations in the values of their failure rate (failure frequency) under the impact of natural and socio-economic factors are revealed. The wavelet spectrums of the failure frequency were constructed, which revealed several historical periods of accidences. It is proposed to consider the failure rate as the output signal of a dynamic system with many difficultly formalized inputs. The stability of this dynamic system is evaluated. The ways of formalizing a multidimensional dynamic model of accidence in main electrical grids of power systems are outlined.
\end{abstract}

\section{Introduction}

It is known that the causes of major accidents in electrical grids with massive damage to overhead lines (OHL), often called extreme climatic conditions. These include elevated ice and rime coatings, hurricanes, natural fires, etc.

Human activity also has a comparable impact on accident rate - unauthorized exposure to overhead line elements and their poor-quality operation: collision of vehicles with towers, trapping wires by hoisting mechanisms, untimely detection defects, etc. Thus, in the general case, the accident rate of overhead lines randomly depends on the impact of natural and social (socio-economic) factors.

In the Table 1 as an example, the structure of stable (i.e. unrecoverable by the autoreclosing), failure of $500 \mathrm{kV}$ overhead lines is given with a total length of about 8.5 thousand $\mathrm{km}$ of the central European part of the country for the period 2011-2018. From table 1 it follows that the social (paragraphs 1,4) and natural influences (paragraphs 2, 3, 5) are approximately equally affect the accident rate of overhead lines.

Earlier in [1], it was noted that the available oscillation periods accidents in electrical grids are close to (quasi) eleven solar activity cycle. The latter definitely affects the change in natural and climatic characteristics (there is an opinion that cycles solar activity have an effect on social phenomena). However, this assumption in [1] was considered as a hypothesis, requiring verification and targeted research. Accident cycles in electric networks were considered in [1] on example of statistical data on unrecoverable failures of $500 \mathrm{kV}$ overhead lines also the central European part of the country for the period 1974-2001. (specific damage was given, 1 / (year $100 \mathrm{~km}$ ), or rather, the average failure rate or the failure frequency $\omega)$. Therefore represented keen interest to extend this time period and clarify the hypothesis of cyclical accidents in the main electrical grids of power systems.

Table 1. Organizational causes of failures for the period 20112018.

\begin{tabular}{|c|c|c|c|}
\hline \multirow[t]{2}{*}{ № } & \multirow[t]{2}{*}{ Failure cause } & \multicolumn{2}{|c|}{$\begin{array}{l}\text { Failure } \\
\text { number }\end{array}$} \\
\hline & & pcs & $\%$ \\
\hline 1 & $\begin{array}{l}\text { Failure to comply with deadlines, } \\
\text { failure to perform the required } \\
\text { amount of maintenance or repair of } \\
\text { equipment and devices, including: }\end{array}$ & 34 & 12.8 \\
\hline 1.1 & $\begin{array}{l}\text { Untimely identification and } \\
\text { elimination defects (broken or } \\
\text { untwining wires and ground wires, } \\
\text { the destruction of the set of } \\
\text { insulators) }\end{array}$ & 17 & 6.4 \\
\hline 1.2 & Other interruptions & 17 & 6.4 \\
\hline 2 & Birds intervention & 5 & 1.9 \\
\hline 3 & $\begin{array}{l}\text { Exceeding the parameters of the } \\
\text { impact of natural disasters regarding } \\
\text { the conditions of the project }\end{array}$ & 8 & 3.0 \\
\hline 4 & $\begin{array}{l}\text { The intervention of unauthorized } \\
\text { persons and organizations not } \\
\text { involved in the technological process }\end{array}$ & 91 & 34.2 \\
\hline 5 & $\begin{array}{l}\text { The effects of recurring natural } \\
\text { disasters, including: }\end{array}$ & 121 & 45.5 \\
\hline 5.1 & Ice and rime coatings & 17 & 6.4 \\
\hline 5.2 & Atmospheric overvoltage (thunder) & 57 & 21.4 \\
\hline 5.3 & Natural fires & 17 & 6.4 \\
\hline 5.4 & $\begin{array}{l}\text { Other effects of adverse natural } \\
\text { events (falling trees) }\end{array}$ & 30 & 11.3 \\
\hline 6 & Undetected reasons & 7 & 2.6 \\
\hline 7 & Total & 266 & 100.0 \\
\hline
\end{tabular}

* Corresponding author: ryabchenko.vn@yandex.ru 


\section{About accidents cyclicality}

Archives for all technological violations of OHL $500 \mathrm{kV}$ of the considered region were raised for the subsequent 2002-2018 In fig. 1 The frequency of unrecoverable OHL failures of $500 \mathrm{kV}$ over 45 years in the interval from 1974 to 2018 is presented with overlaying four peaks held eleven-year cycles of solar activity (from the 21 st to the 24th, the last cycle according to the reported data had two bursts: 2013 and 2014); the peak of the 25 th cycle is expected in 2023.

As can be seen from fig. 1, the values of the failure frequency are oscillatory in nature, varying in a wide range: from 0.111 (year $100 \mathrm{~km}$ ) to 1980 and 2018 up to $0.861 /($ year $100 \mathrm{~km}$ ) in 1998 (Historical note: in 1980, the summer Olympic Games were held in our country, in 2018 - soccer world cup, and in 1998 - was declared default).

In years of the highest solar activity (Fig. 1), there is a slight increase in the OHL failure frequency. However, in between practically of each cycle, bursts of OHL accidence are found that exceed those for the peaks of solar activity. For example - this is earlier mentioned 1998 or 2010 with its most severe fires in the European part of the country as a result of an abnormally hot summer.

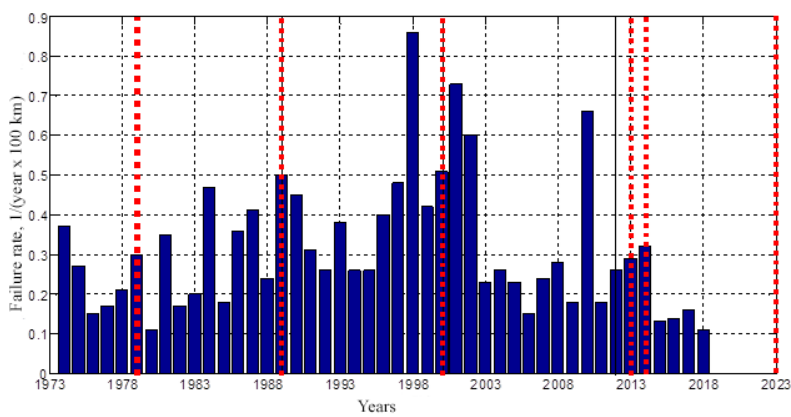

Fig. 1 - Values of the failure rate of $500 \mathrm{kV}$ overhead lines for the period 1974-2018 with solar activity cycles (dashed)

Fig. 1 is an amplitude-time representation parameter (signal). But, as you know, no less significant information hiding in its frequency domain. For this purpose, the graph in Fig. 1 was subjected to the socalled wavelet analysis [2], which allowed the timefrequency separation of the process in Fig. 1.

Figure 2 shows the wavelet spectrum of the failure rate with the Haar mother wavelet. By the way, almost identical given in fig. 2 wavelet spectra obtained using as mother wavelets of Daubechies (db), Koifman (coif), simlet (sym), biorthogonal spline wavelet (bior) and reverse biorthogonal spline wavelet (rbio).

In the behavior of the failure rate in Fig. 2 distinguishable three time periods from the standpoint of the intensity (disturbance) of its frequency spectrum: from 1974 to the beginning of the 1990s; since the early 1990s before early 2000s and since the beginning of the 2000s. Until now. The first period has an unperturbed, relatively uniform, calm frequency spectrum. The second cycle is the spectral "storm". Finally the third period - stabilization and calming of the frequency spectrum; some the surge in intensity is apparently associated with the events of 2010 (see above). It must be assumed that Fig. 2 reflected events that occurred in our country. In particular, the second period is the time of decay once a single country and the collapse of its economy.

Based on the analysis of events in Fig. 1 and 2 does not seem possible to confirm the attractive hypothesis expressed in [1], that the available periods of fluctuations of accidence in electrical networks close to the eleven-year cycle of solar activity. This is too simple an explanation of the processes in the issue of reliability.

Cause-and-effect links are much more complicated. Their resulting exposure is determined by multifactorial and difficult to formalize addition of environmental influences and socio-economic relationship. However, regardless of this, the failure rate under consideration is not a set of fixed values that depend, for example, on the material of the towers or the nominal line voltage, and a dynamic process with a change in characteristic time periods generated by some dynamic system.

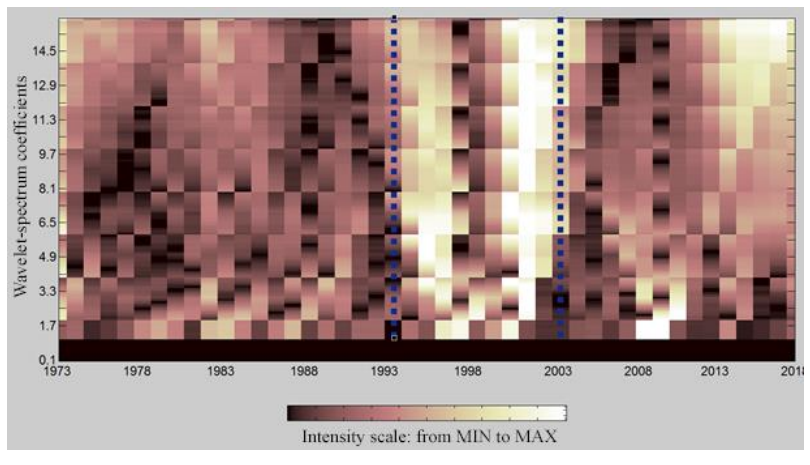

Fig. 2 - Wavelet spectrum of the failure rate of a $500 \mathrm{kV}$ overhead line

As is known, mathematical models of dynamical systems are conventionally divided into two disjoint classes: autonomous and input-output [3]. In the first case, the output signals of the system are generated by internal state transitions under the action of nonzero initial conditions. Moreover, all non-formalized external influences of this system are converted into equivalent initial conditions [3]. In the second case, the system output signals are a homomorphic (not one-to-one) transformation of the corresponding input signals.

In our case, we formulate a particular problem: which dynamic system reproduces the sequence of output signals with the smallest possible deviations from the observed series in the form of failure rate (Fig. 1)?

In this case, an identifiable model should be suitable for analysis of its stability. (Not strictly speaking the steady movement of a dynamic system is determined by two trends that depend on the physical nature of the process: conservative, seeking to compensate for external influences, resisting these influences and leading, as a rule, to oscillatory motion, and dissipative, dissipating the energy of these oscillations.) We formulate the calculated conditions for the model of the dynamic system under study: 
- It belongs to the class of discrete dynamic systems [3], since changes in the failure rate occur discretely, with a step of "integration" of one year;

- It is positive (positive, non-negative), i.e. generates only positive values of the output signal, since the parameter of the failure rate is at least a non-negative value [4];

- it can be represented in the state space, i.e. is described a set of physical or abstract variables characterizing the behavior of the system in the future, provided that the state at the present moment of time is known [3];

- It possesses the property of non-stationarity, as its parameters change in time, from step to step;

- It considered as an autonomous system, as in this case there is no additional information that could be taken as input;

- It's limited to the set of data analyzed, starting in 2002 - see the wavelet spectrum in Fig. 2. Otherwise, the identification results will be significantly affected by the so-called "tails" data relating to previous historical periods [5].

We can realize Identification of the model for changing the failure rate per 2002-2018 in the class of positive, discrete, non-stationary, autonomous dynamical systems in the state space, on the base of transformations of the Hankel matrix (a square matrix with all diagonals perpendicular to the main equal elements formed from a data array) using the least squares method [5]; we obtain the following discrete equations:

$$
\mathbf{x}(t+1)=\boldsymbol{A}(t) \mathbf{x}(t), \omega(t)=\mathbf{c}^{\mathrm{T}} \mathbf{x}(t)
$$

Where $\mathbf{x}(t)$ - state vector, $\omega(t)$ - system's output signal (failure rate), $\mathbf{c}^{\mathrm{T}}=\left[\begin{array}{lll}1 & 0 & 0\end{array}\right]$.

$$
\mathbf{x}(1)=\left[\begin{array}{l}
\omega_{2002} \\
\omega_{2003} \\
\omega_{2004}
\end{array}\right], \mathbf{x}(2)=\left[\begin{array}{l}
\omega_{2003} \\
\omega_{2004} \\
\omega_{2005}
\end{array}\right], \ldots, \mathbf{x}(N)=\left[\begin{array}{l}
\omega_{2016} \\
\omega_{2017} \\
\omega_{2018}
\end{array}\right]
$$

- values of the state vector at the corresponding step of "integration"; $t=1,2, \ldots, \mathrm{N} . \mathrm{N}$ is the interval of "integration" (in this case, $\mathrm{N}=15$ );

$$
\begin{gathered}
A(1)=\left[\begin{array}{ccc}
0 & 1 & 0 \\
0 & 0 & 1 \\
0,4655 & 0,0621 & 0,2404
\end{array}\right], \\
\operatorname{eig} \boldsymbol{A}(1)=\{-0,33264 \pm j 0,6439 ; 0,8933\} \\
\boldsymbol{A}(N)=\left[\begin{array}{ccc}
0 & 1 & 0 \\
0 & 0 & 1 \\
0,4125 & 0,2326 & 0,1548
\end{array}\right], \\
\operatorname{eig} \boldsymbol{A}(N)=\{-0,3773 \pm j 0,5579 ; 0,9093\}
\end{gathered}
$$

- matrices of eigen (free) dynamics in the steps of "integration" and the corresponding sets of eigenvalues (poles).

The distribution of poles (eigA eigenvalues) of the identified failure rate model (1) - (3) for the period 2002- 2018 years presented in fig. 3 .

Since for the asymptotic stability of a discrete dynamical system it's necessary and sufficient that the location of its poles on the complex plane $\mathrm{C}$ inside the unit circle, centered at the beginning of $\mathrm{C}$, then from the diagram in Fig. 3 that the identified model is asymptotically stable [3] or, in other words - Shurov model. In this case, the margin (radius, degree) of stability of the indicated model of the failure rate is shown in Fig. 4.

We recall that in the case of a discrete dynamic system (1), the stability margin is calculated as follows:

$$
\rho_{\text {st }}=\left|\operatorname{Re} \lambda_{\text {max }}\right|<1
$$

The stability margin is an extremely important characteristic of a dynamic system and characterizes the attenuation rate of transients in it. Moreover, the lower $\rho_{\text {st }}$, the closer the transitional oscillation in the system to aperiodic and rapidly decaying processes.

However, the presence of one or another margin of stability in a dynamic system is not a guarantee that sufficiently small perturbations will not lead to a loss of stability. Therefore, we consider the $\varepsilon$-spectrum [6] in as a method for analyzing robustness of stability (rudeness - freedom from influence of disturbances) of the dynamic model of the failure rate. This method operates with complex numbers $z_{i}$ that make up the set of the following form ( $\varepsilon$-spectrum):

$$
\operatorname{eig}_{\varepsilon}(\boldsymbol{A})=\left\{z_{i}: \sigma_{\min }\left(z_{i} \boldsymbol{E}-\boldsymbol{A}\right) \leq \varepsilon\right\}
$$

In formula (5), $\sigma_{\min }$ is the minimum singular number of the matrix $z_{i} \boldsymbol{E}-\boldsymbol{A} . \quad \varepsilon$ is the given positive number (tolerance), $\mathrm{E}$ is the identity matrix. In other words, the $\varepsilon$-spectrum is the set of complex numbers $\mathrm{z}$ such that all the matrices $z \boldsymbol{E}-\boldsymbol{A}$ have minimal singular numbers that do not exceed some finite number (tolerance) $\varepsilon$ that characterizes these matrices as "close to degenerate" or "practically degenerate"

Stability analysis of the failure rate model for period 2002-2018 based on the $\varepsilon$-spectrum (Fig. 5) gives an estimate of a small degree of robustness, lying in the range $(0,75 \ldots 1,25) \times 10^{-1}$. In other words, the stability of this model is subject to significant the influence of relatively small perturbations of the elements of matrix A.

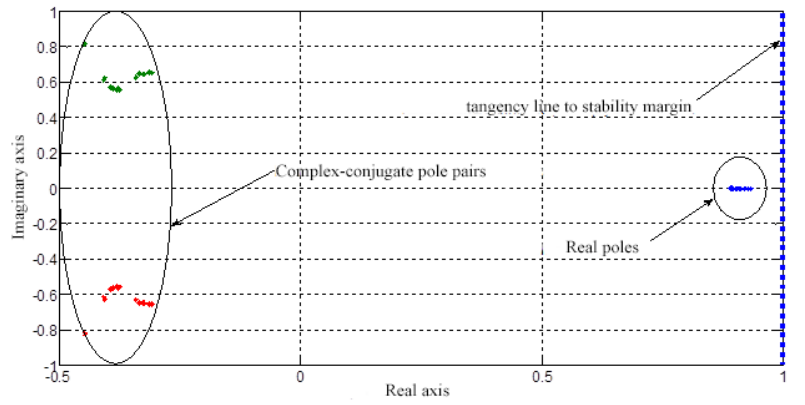

Fig. 3 - Poles of the model of the failure rate of $500 \mathrm{kV}$ overhead lines for the period 2002-2018 years in the state space 


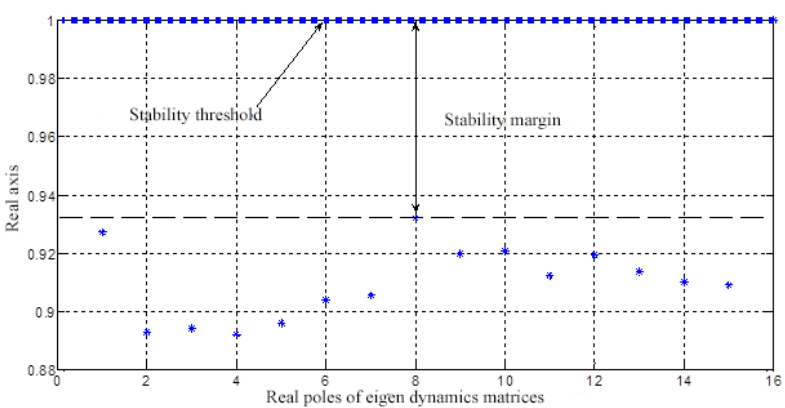

Fig. 4 - Stability margin of a discrete non-stationary dynamic failure rate model

This estimate is obtained by numerically analyzing regions on the complex plane in which the poles (eigenvalues) of all matrices $A$ of the model of the dynamical system (1) obtained at relatively close but different initial approximations are localized.

In this sense, the $\varepsilon$-spectrum method is regular with respect to the used strategy of "weighting" (increasing the intensity of disturbances), i.e. guarantees the independence of the obtained stability margin estimates from the choice of initial approximations and the "weighting" strategy.

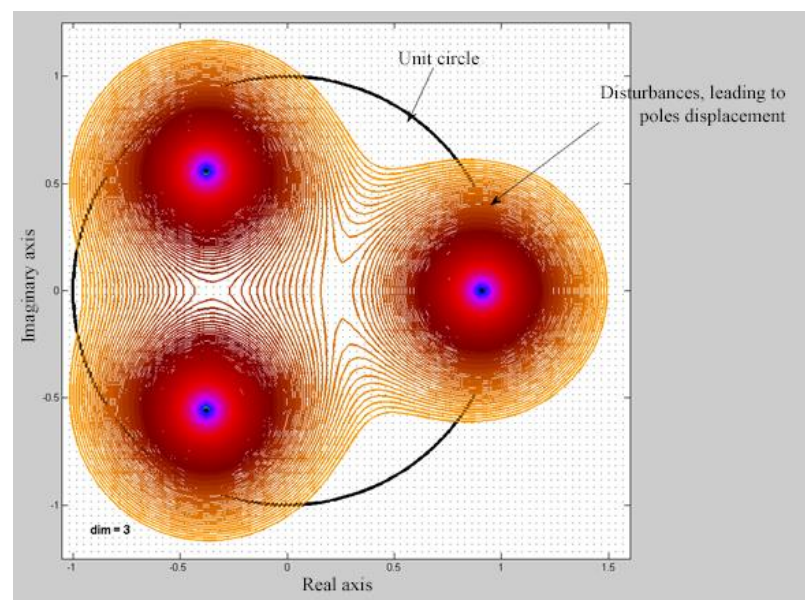

Fig. 5 - Evaluation of the robustness of the stability margin of the failure flow parameter model $\varepsilon$-spectrum based (two-dimensional looking)

Thus, the dynamic model of the $500 \mathrm{kV}$ OHL failure rate is stable over the considered time period, but is subject to the influence of relatively small perturbations. We relate this theoretical assumption to current practice of operation of main electrical grids.

Figure 6 shows the age characteristics of the OHL of $500 \mathrm{kV}$ in the region under consideration. As you can see, about $40 \%$ of OHL of $500 \mathrm{kV}$ is older than 50 years, and lines "younger" 30 years, i.e. built after the events of the 90 s last century - less than $20 \%$.

According to $[7,8]$, there is a noticeable increase in the accident rate of the deterioration character of overhead lines of all classes stress due to mass aging the country. However, the evidence on fig. 1 (if you do not take into account the anomalous 2010) indicates on the contrary: the reliability of the $500 \mathrm{kV}$ OHL is continuously improving despite the fact that there is almost no new construction or reconstruction of them. A possible explanation for this paradox is as follows.

An analysis of the reliability characteristics of the overhead lines of the Unified National Power Grid revealed [9] that over the past 30 years, the average duration of scheduled overhead lines repair in the main electrical grids of power systems has grown from 12-17 to $95-149$ hours, i.e. almost 10 times.

At the same time, the main part of deliberate outages is associated with repairs or maintenance of the line itself, and not with its reconstruction or other external reasons.

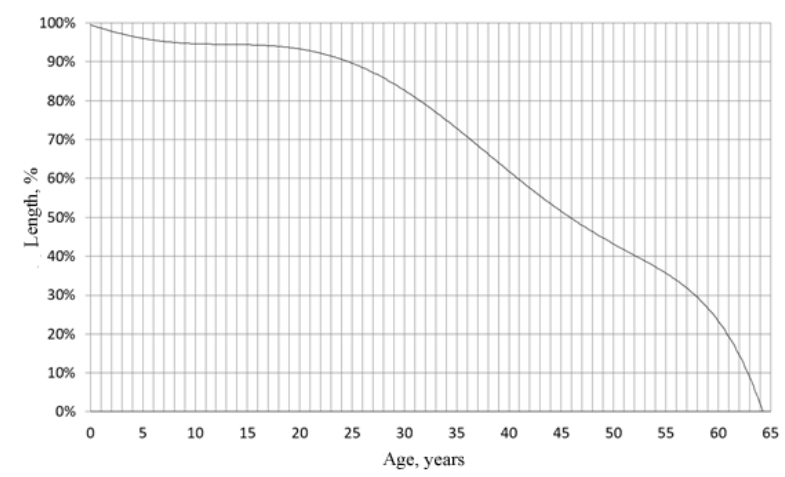

Fig. 6 - cumulative probability distribution function of age characteristics of $500 \mathrm{kV}$ OHL

In other words, maintaining the operability of morally and physically worn out grid elements is provided not by reconstruction, but by repairs. It was on this model of management that domestic energy systems were forced to work in the 90 s of the last century in the conditions of the collapse of the economy.

Thus, in spite of the favorable reliability characteristics of the $500 \mathrm{kV}$ overhead lines in Fig. 1, it is hardly possible to speak of a favorable prognosis of their accident rate.

\section{Conclusions}

1. Overhead lines of the Unified National Power Grid form the backbone of energy systems, while being the most damaged elements. Therefore, accident risks are traditionally given close attention.

2. Over the past decades, significant fluctuations in the failure rate of high-voltage lines of $500 \mathrm{kV}$ under the influence of natural and socio-economic factors were revealed: from 0.11 to $0.861 /($ year $100 \mathrm{~km})$. Moreover, the previously stated hypothesis is not confirmed that the available periods of accident fluctuations in electric networks are close to the eleven-year cycle of solar activity.

3. Using wavelet analysis, the presence in the frequency spectrum of the failure rate of a $500 \mathrm{kV}$ overhead line of three distinct time periods, approximately corresponding to the socio-economic changes in the country, is shown. This once again confirms that the parameter under consideration is determined by the multifactorial and difficult to formalize addition of environmental influences and socio-economic relations. 
4. Based on the oscillatory process of changing the failure rate of a $500 \mathrm{kV}$ overhead lines, this parameter is described as an output signal in the class of positive discrete non-stationary autonomous dynamic systems in the state space.

5. The asymptotic stability of this dynamical system is investigated. The system was steady. However, the robustness of the model of the failure rate of a $500 \mathrm{kV}$ overhead lines turned out to be insufficiently favorable in terms of reliability. That is, its stability is significantly affected by relatively small perturbations.

6. The results of mathematical modeling and the corresponding conclusions regarding insufficient robustness are indirectly confirmed by an analysis of the age structure of the $500 \mathrm{kV} \mathrm{OHL}$ and change in their reliability characteristics in terms of repair and maintenance services in the direction of their deterioration.

\section{References}

1. V. Skopintsev The quality of electric power systems: reliability, safety, efficiency, survivability (Energoatomizdat, 2009)

2. S Malla Wavelets in Signal Processing (Mir, 2005) 3. T. Kailath Linear Systems (Prentice Hall, Englewood Cliffs, NJ. 1980)

4. W. Haddad, V. Chellaboina, Q. Hui Nonnegative and compartmental dynamical systems (Princeton University Press, 2010)

5. Van Overschee P., de Moor B.L. Subspace identification for linear systems: Theory, Implementation, Applications (Springer Science and Business Media, 2012)

6. L.N. Trefethen, M. Embree Spectra and pseudospectra. (Princeton Univ. Press, 2005)

7. R. Kaverina, F Kogan, L. Yakovlev, Improving the reliability of overhead lines $35-750 \mathrm{kV}$. General issues of OHL condition. Novosti Electrotehiki, № 4(46) (2007)

8. R. Kaverina, F Kogan, L. Yakovlev Improving the reliability of overhead lines $35-750 \mathrm{kV}$. Reliability of wires and lightning protection cables. Novosti Electrotehiki, № 5(47) (2007)

9. A. Abdurahmanov, S. Glushkin, I. Protasenko, A. Shuntov About reliability characteristics of OHL in electrical power grids. Electrichestvo, № 8, p. 12-17 (2018) 